\title{
Molecular Imaging-Guided Theranostics and Personalized Medicine
}

\author{
Hong Zhang, ${ }^{1}$ Mei Tian, ${ }^{2}$ Enzhong Li, ${ }^{3}$ Yasuhisa Fujibayashi, ${ }^{4}$ \\ Lie-Hang Shen, ${ }^{5}$ and David J. Yang ${ }^{2}$ \\ ${ }^{1}$ Department of Nuclear Medicine, Second Affiliated Hospital of Zhejiang University School of Medicine, 88 Jiefang Road, \\ Zhejiang, Hangzhou 310009, China \\ ${ }^{2}$ Department of Experimental Diagnostic Imaging, University of Texas MD Anderson Cancer Center, 1515 Holcombe Boulevrd, \\ P.O. Box 59 Houston, TX 77030, USA \\ ${ }^{3}$ Department of Health Sciences, National Natural Science Foundation of China, Beijing, China \\ ${ }^{4}$ National Institute of Radiological Sciences, Chiba, Japan \\ ${ }^{5}$ Isotope Application Division, Institute of Nuclear Energy Research, 1000, Wenhua Road, Jiaan Village, Taoyuan County, \\ Longtan Township 32546, Taiwan
}

Correspondence should be addressed to Hong Zhang, dyang@mdanderson.org

Received 20 May 2012; Accepted 20 May 2012

Copyright (c) 2012 Hong Zhang et al. This is an open access article distributed under the Creative Commons Attribution License, which permits unrestricted use, distribution, and reproduction in any medium, provided the original work is properly cited.

Molecular imaging agents and a parallel progress in instrumentation of imagining technology have demonstrated to be effective in improving diagnosis, prognosis, planning, and monitoring of personalized medication. Molecular imaging modalities include positron emission tomography (PET), single photon emission computed tomography (SPECT), magnetic resonance imaging (MRI), computed tomography (CT), ultrasound (US), and optical (Raman, quantum dots, bioluminescence). Among these imaging modalities, PET and SPECT agents could provide target specific information as well as function, pathway activities, and cell migration in the intact organism. Furthermore, the radiotracer could noninvasively assess diseases treatment endpoints which used to rely almost exclusively on biopsies and histopathological assays. New leads on the development of personalized theranostic (image and treat) agents would allow more accuracy in the selection of patients who may respond to treatment.

Topics covered in this special issue include advances in biomarkers in preclinical drug discovery, PET/CT and SPECT/CT in disease management, image-guided therapy approach of diseases, imaging technology in drug development, and progress in instrumentation of imaging technology. For instance, upregulation of transporter expressions has often been observed in tumor cells to facilitate their accelerated rates of uptake. Advances in biomarkers in transporter system-based imaging in oncology and neurological diseases such as amino acid (FL. Kong et al.), glucose (YH. Zhang et al.) and dopaminergic (LH. Shen et al.; HF. Hou et al.) transporters were reported. These biomarkers showed that specific uptake in lesions compared to clinic imaging agent. In addition, these biomarkers were capable to be labeled with theranostic isotopes for personalized medication.

Most tumors have a considerable proportion of hypoxic cells that are resistant to radio/chemotherapy, with a high propensity to metastasize, and result in worse therapeutic outcome. The contribution by M. Ali et al. and H. Fuji report their newly developed ${ }^{99 \mathrm{~m}} \mathrm{Tc}$-N4-NIM and ${ }^{125}$ I-IPOS probes for tumor hypoxia. The preclinical findings showed that these biomarkers could image tumor hypoxia by SPECT. The ability to quantify tissue hypoxia would allow the physicians to select patients for additional or alternative treatment regimens that would circumvent the ominous impact of hypoxia. Along this line, L. Jiang and coworkers report the peptide based radiotracer targeting tumor angiogenesis via VEGF integrin alpha(v) beta3. J. Sims-Mourtada and coworkers report ${ }^{131}$ I-labeled derivatives of the sonic hedgehog $(\mathrm{HH})$ protein for detection of cancer cells via $\mathrm{HH}$ receptors. Molecular imaging of cancer stem cell trafficking was reported by T. Xia and co-workers.

Hybrid molecular imaging modalities (PET/CT, SPECT/ $\mathrm{CT}$ ) provide high-sensitivity functional and high resolution 
anatomical imaging, which are important in design personalized treatment. To avoid radiation exposure from multiple slices-CT, K. Tang and co-workers report a reduction of CT tube voltage from $120 \mathrm{kv}$ to $80 \mathrm{kv}$, the radiation dose could be reduced by $32-42 \%$ without losing low-contrast detectability. F. Chao and $\mathrm{H}$. Zhang report the value of using PET/CT in the staging of nonsmall cell lung cancer. $\mathrm{Y}$. $\mathrm{Hu}$ and co-workers report the application of PET/CT in assessment of treatment outcome. The application of SPECT/CT in the differential diagnosis of bone metastasis (Y. Zhang et al.) and Parkinson's disease (L. Wang et al.) was reported. Miyake $\mathrm{K}$ and co-workers report the application of FDG, MET, and FLT-PET/CT for the management of gliomas. L. Yang and co-workers report the value of using myocardial contrast echocardiography quantitative analysis during adenosine stress over visual analysis before therapy in acute coronary pain. The quantitative analysis correlates well with thrombosis in myocardial infarction.

In summary, molecular imaging could integrate metabolomics and chemical biology. Molecular imaging agents could characterize target expressions, understand the disease progression, prediction for drug response and toxicity, staging, grading, and micrometastasis, and support animal studies. This special issue provides a platform of efficacy of personalized medication from molecular imaging technology which may have high impact on drug discovery, delivery, and development.

\author{
Hong Zhang \\ Mei Tian \\ Enzhong $L i$ \\ Yasuhisa Fujibayashi \\ Lie-Hang Shen \\ David J. Yang
}




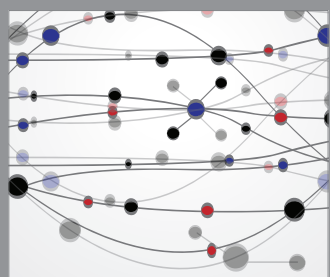

The Scientific World Journal
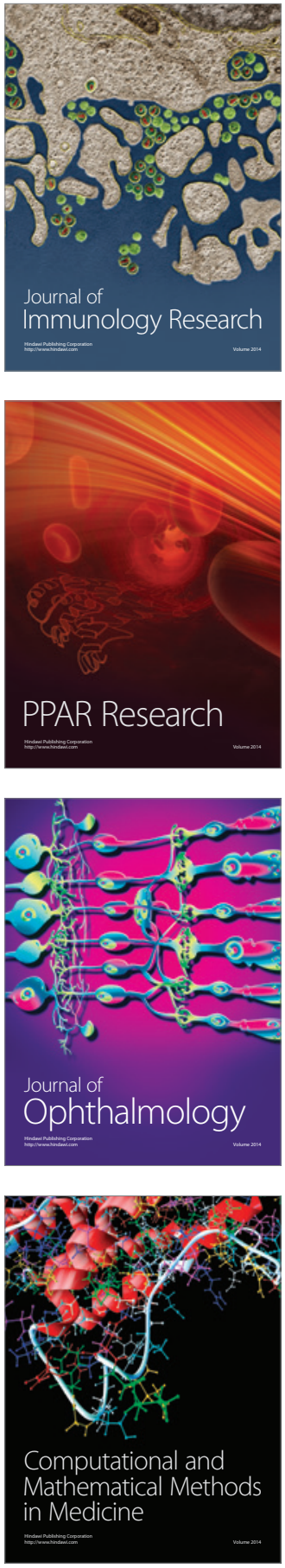

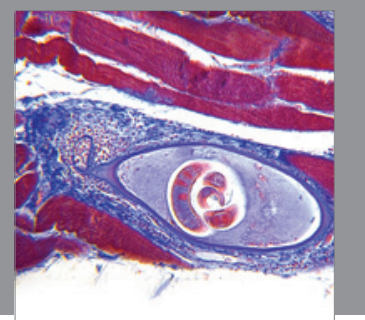

Gastroenterology

Research and Practice
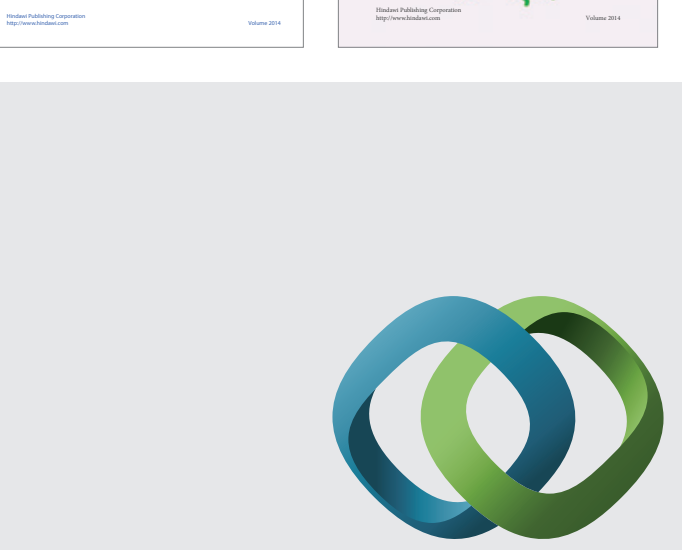

\section{Hindawi}

Submit your manuscripts at

http://www.hindawi.com
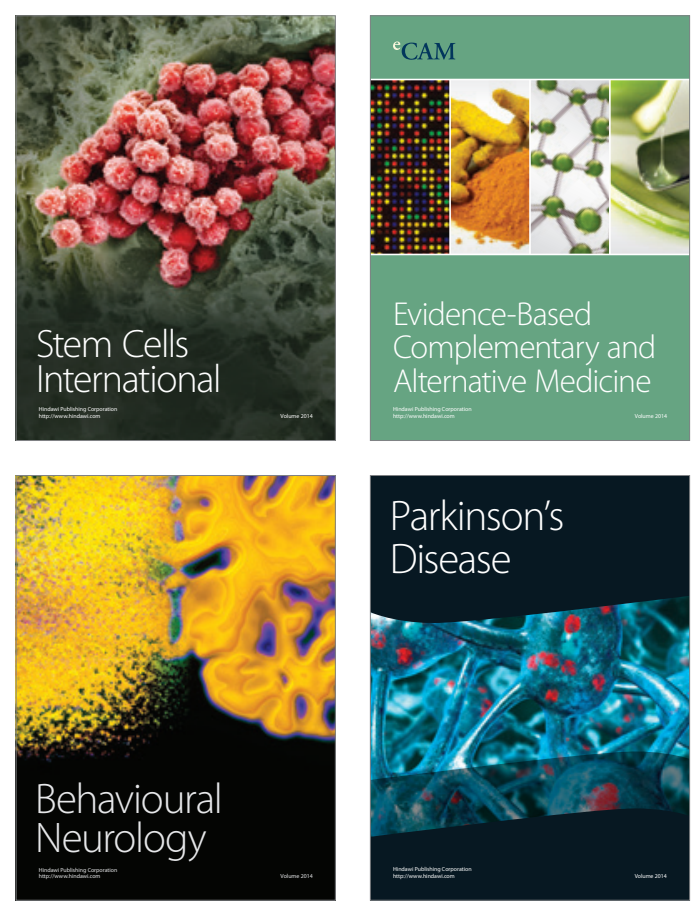

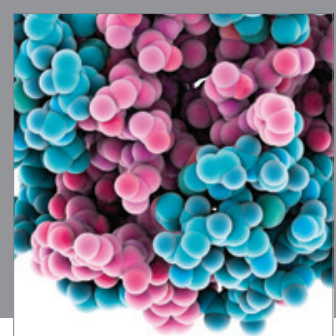

Journal of
Diabetes Research

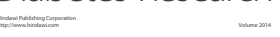

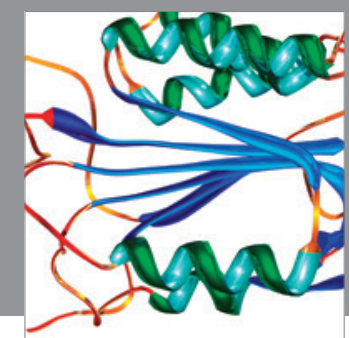

Disease Markers
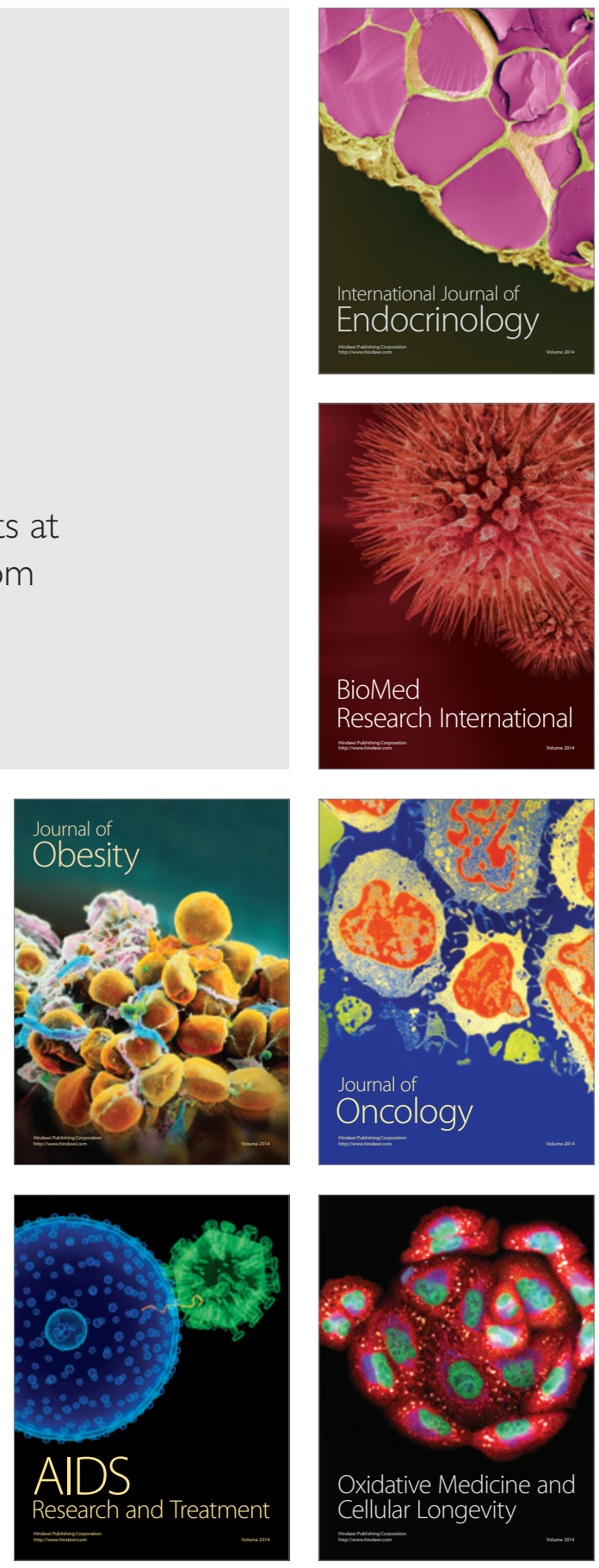